\title{
Manejo de residuos sólidos y el riesgo laboral en el hospital Víctor Ramos Guardia - Huaraz, 2013
}

\author{
Solid waste management and labor risk at Víctor Ramos Guardia \\ Hospital - Huaraz, 2013 \\ Rafael Norabuena Penadillo ${ }^{1}$, Arturo Tuya Ordóñez ${ }^{1}$, Elizabeth Vélez Salazar ${ }^{1}$ \\ y Julio Menacho López ${ }^{1}$
}

\section{RESUMEN}

Analiza la relación entre el manejo de residuos sólidos y el riesgo laboral al que están expuestos los trabajadores de salud del hospital Víctor Ramos Guardia (VRG), sobre la base de la norma técnica de gestión y manejo de residuos sólidos del Ministerio de Salud. Para ello, se realizó un estudio descriptivo correlacional, transversal, donde la muestra estuvo conformada por 87 trabajadores asistenciales del hospital VRG de Huaraz, a quienes se les aplicó una encuesta y además se empleó una guía de observación. Los resultados revelan que, el manejo interno de los residuos sólidos es aceptable (46\%), esto se corrobora al relacionar el manejo de residuos sólidos catalogados como aceptable con el riesgo laboral que fue considerado como mediano. El 32,2 \% de los trabajadores están expuestos a un peligroso alto riesgo durante sus actividades diarias, existiendo un preocupante 29,1\% de trabajadores que han sufrido pinchazos como parte de su labor. El almacenamiento primario, intermedio y central de dichos residuos fueron calificados como deficientes, existiendo graves problemas en la segregación que fue evaluado como muy deficiente. Se concluyó que existe una relación significativa entre el manejo inadecuado de residuos sólidos y el riesgo laboral al que están expuestos los trabajadores de salud del hospital Víctor Ramos Guardia.

Palabras clave: residuos sólidos; riesgo laboral; segregación de residuos sólidos.

\section{ABSTRACT}

Analyze the relationship between solid waste management and occupational risk to which the health workers of the Víctor Ramos Guardia hospital (VRG) are exposed

1 Universidad Nacional Santiago Antúnez de Mayolo. Huaraz, Perú. 
based on the technical standard for solid waste management Of the Ministry of Health. A cross - sectional, correlational descriptive study was carried out, where the sample consisted of 87 care workers from the VRG hospital in Huaraz, to whom a survey was applied. An observation guide was used to evaluate the solid waste. Among the most relevant results are the solid waste internal management is acceptable (46\%), this is corroborated by the relation of the solid waste management classified as acceptable with the occupational risk that was considered as medium. $32.2 \%$ of these workers are exposed to a dangerous high risk during their daily activities, with a worrying $29.1 \%$ of workers who have suffered a puncture. The primary, intermediate and central storage of these was classified as deficient, with serious problems in the segregation that was considered very deficient. It was concluded that there is a significant relationship between the inadequate solid waste management and the occupational risk to which the health workers of hospital Victor Ramos Guardia are exposed.

Keywords: solid waste; occupational risk; segregation of solid waste.

\section{INTRODUCCIÓN}

Los residuos sólidos generados en los hospitales y otras instituciones sanitarias generan riesgos para la salud fundamentalmente por el carácter infeccioso de algunos componentes. Aumentan tales riesgos la presencia frecuente de objetos punzo-cortantes y la presencia eventual de cantidades menores de sustancias tóxicas, inflamables y radioactivas de baja intensidad. La Organización Mundial de la Salud (2013) indica que las actividades de la salud generan el $85 \%$ de residuos comunes y $15 \%$ de material peligroso, administrándose cada año en todo el mundo 16.000 millones de inyecciones, cuyas agujas usadas, no se desechan correctamente. En el año 2010, las inyecciones con jeringas contaminadas causaron: 1,7 millones de Hepatitis B y 33.800 infecciones por el VIH.

En ese sentido, Ortiz (2010) indica que, en el estudio realizado por el Servicio de Salud Pública de los Estados Unidos, en el 2010, se concluyó que el manejo inapropiado de los desechos sólidos hospitalarios está relacionado con la generación de 22 enfermedades infecciosas comunes. Es decir que el personal asistencial de los establecimiento de salud y servicios médicos de apoyo (médicos, obstetras, enfermeras, tecnólogos médicos y técnicos, de enfermería) de todos los Centros Asistenciales de Salud están en riesgo de sufrir algún daño potencial como consecuencia de la exposición o contacto a residuos peligrosos; destacándose los residuos punzo cortantes como los principalmente implicados en los accidentes en trabajadores de salud y en la transmisión de enfermedades infecciosas. Algunas de estas lesiones exponen a los trabajadores a patógenos contenidos en la sangre que pueden trasmitir infecciones tales como la hepatitis B, la hepatitis C, y la contaminación con el Virus de la Inmunodeficiencia Humana (VIH), 
entre otros. Las infecciones producidas por cada uno de estos patógenos pueden poner en peligro la vida, pero son prevenibles si se toman acciones ante ello.

En el Perú, se ha establecido la norma técnica sobre procedimientos para el manejo de residuos sólidos hospitalarios emitida por el Ministerio de Salud (MINSA), aprobada con resolución $\mathrm{N}^{\circ}$ 217-2004/MINSA, donde se enfatiza que todo establecimiento de salud, debe implementar un sistema de gestión para el manejo de residuos sólidos hospitalarios (MINSA 2004), en tal sentido el hospital Víctor Ramos Guardia (VRG) de la ciudad de Huaraz está en la obligación de implementar y adaptar dicha norma técnica para prevenir, controlar y minimizar los riesgos sanitarios y laborales de los residuos hospitalarios, así como el impacto negativo en la salud pública y el medio ambiente. Sin embargo, en el nosocomio huaracino, no se cuenta con un diagnóstico actualizado del manejo de residuos sólidos y de los riesgos que pueda causar en el personal de salud, a partir de lo cual la presente investigación tiene como objetivo desarrollar ese análisis, recopilando información sobre la situación actual de la problemática, contribuyendo en mejorar la gestión de los residuos hospitalarios peligrosos, así como la evaluación de los riesgos laborales, que ponen en peligro la salud de los trabajadores.

Por lo tanto, seguidamente se presenta toda la información recopilada de los trabajadores del hospital Víctor Ramos Guardia de Huaraz y el análisis correspondiente de todos los datos que conllevan a conclusiones importantes que han permitido proponer recomendaciones y la elaboración de un plan de mejora para el manejo adecuado de los residuos sólidos del hospital Víctor Ramos Guardia de la ciudad de Huaraz (VRG), así mismo contribuir a prevenir enfermedades y la protección del medio ambiente.

\section{MATERIALES Y MÉTODOS}

En concordancia con el problema planteado y los objetivos de la investigación, el presente estudio descriptivo, correlacional transversal, de diseño no experimental, incluyó como muestra a 87 trabajadores asistenciales del hospital Víctor Ramos Guardia de Huaraz. (32 enfermeras, 07 obstetrices, 03 tecnólogos médicos, 04 técnicos de laboratorios, 36 técnicos de enfermería, y 05 trabajadores de servicio), para lo cual se aplicó el muestreo probabilístico (aleatorio simple).

Para relacionar el manejo interno de residuos sólidos y el riesgo laboral se aplicó una encuesta estructurada dirigida al personal asistencial, así mismo se usó una guía de observación no participativa, empleando como instrumento un cuestionario estructurado y una lista de cotejo que estuvieron basadas en la norma técnica "gestión y manejo de residuos sólidos en establecimientos de salud y servicios médicos de apoyo”.

Para la inclusión en el grupo de muestra se consideró como requisito previo la firma voluntaria de la declaración de consentimiento informado de los participantes, respetándose en todo momento el principio de autonomía y responsabilidad individual, así como la privacidad y la confiabilidad de la información. 
La validez del instrumento se realizó mediante juicio de expertos, con una confiabilidad del 0,879 en el alfa de Cronbach, con lo cual el instrumento utilizado fue confiable, para la correlación de las variables se usó el coeficiente de correlación de Spearman obteniéndose una correlación positiva considerable. Para el procesamiento de la información se usó el programa estadístico SPSS V 19.0, utilizando como instrumento de análisis las tablas.

\section{RESULTADOS}

Tabla 1. Calificación del manejo interno de los residuos sólidos, hospital Víctor Ramos Guardia, Huaraz, 2013

\begin{tabular}{ccc}
\hline $\begin{array}{c}\text { MANEJO INTERNO DE RESI- } \\
\text { DUOS SÓLIDOS }\end{array}$ & $\mathrm{N}^{\circ}$ & $\%$ \\
\hline Satisfactorio & 28 & 32,2 \\
Aceptable & 40 & 46 \\
Deficiente & 15 & 17,2 \\
Muy Deficiente & 4 & 4,6 \\
\hline TOTAL & 87 & 100
\end{tabular}

En la tabla 1, se evidencia que en el $46 \%$ de trabajadores asistenciales del hospital Víctor Ramos Guardia, califica de aceptable el manejo interno de los residuos sólidos en el nosocomio; resultando preocupante que existe un significativo 17,2\% que le atribuye deficiencias al manejo de dichos residuos.

Tabla 2. Riesgo laboral al que están expuestos los trabajadores de salud y de limpieza, hospital Víctor Ramos Guardia, Huaraz, 2013

\begin{tabular}{ccc}
\hline RIESGO LABORAL & $\mathrm{N}^{\circ}$ & $\%$ \\
\hline Alto riesgo & 28 & 32,2 \\
Mediano riesgo & 45 & 51,7 \\
Bajo riesgo & 14 & 16,1 \\
\hline TOTAL & 87 & 100 \\
\hline
\end{tabular}


En la tabla 2, se observa que un poco más de la mitad de los trabajadores de salud (51,7 $\%$, tienen un riesgo laboral medio en el hospital Víctor Ramos Guardia con respecto a la manipulación de residuos sólidos; mientras que un 32,2 \% de dichos trabajadores indica que están expuestos a un peligroso alto riesgo durante sus actividades diarias, no existiendo las medidas de protección necesarias para su manejo.

Tabla 3. Tipo de accidentes laborales según personal y/o trabajador de salud, hospital Víctor Ramos Guardia, Huaraz, 2013

\begin{tabular}{|c|c|c|c|c|c|c|c|c|c|c|c|c|c|c|}
\hline \multirow{3}{*}{$\begin{array}{c}\text { TIPO DE ACCI- } \\
\text { DENTES LABO- } \\
\text { RALES }\end{array}$} & \multicolumn{12}{|c|}{ PERSONAL Y/O TRABAJADOR DE SALUD } & \multirow{2}{*}{\multicolumn{2}{|c|}{ TOTAL }} \\
\hline & \multicolumn{2}{|c|}{ Enfermeros } & \multicolumn{2}{|c|}{ Obstetras } & \multicolumn{2}{|c|}{$\begin{array}{l}\text { Tecnólogo } \\
\text { Médico }\end{array}$} & \multicolumn{2}{|c|}{$\begin{array}{c}\text { Téc. } \\
\text { Enfermería }\end{array}$} & \multicolumn{2}{|c|}{$\begin{array}{l}\text { Téc. Labora- } \\
\text { torio }\end{array}$} & \multicolumn{2}{|c|}{$\begin{array}{l}\text { Trabajador } \\
\text { de Limpieza }\end{array}$} & & \\
\hline & $\mathrm{N}^{\circ}$ & $\%$ & $\mathrm{~N}^{\circ}$ & $\%$ & $\mathrm{~N}^{\circ}$ & $\%$ & $\mathrm{~N}^{\circ}$ & $\%$ & $\mathrm{~N}^{\circ}$ & $\%$ & $\mathrm{~N}^{\circ}$ & $\%$ & $\mathrm{~N}^{\circ}$ & $\%$ \\
\hline Salpicaduras & 10 & 6,8 & 4 & 2,7 & 4 & 2,7 & 20 & 13,5 & 4 & 2,7 & 7 & 4,7 & 49 & 33,1 \\
\hline Pinchazos & 8 & 5,4 & 4 & 2,7 & 4 & 2,7 & 15 & 10,2 & 4 & 2,7 & 8 & 5,4 & 43 & 29,1 \\
\hline $\begin{array}{c}\text { Contacto con heridas } \\
\text { abiertas }\end{array}$ & 2 & 1,4 & 1 & 0,6 & 0 & 0 & 5 & 3,4 & 0 & 0 & 0 & 0 & 8 & 5,4 \\
\hline Cortaduras & 12 & 8,1 & 2 & 1,4 & 4 & 2,7 & 4 & 2,7 & 0 & 0 & 3 & 2 & 25 & 16,9 \\
\hline Rasguños & 4 & 2,7 & 0 & 0 & 1 & 0,7 & 2 & 1,3 & 1 & 0,7 & 0 & 0 & 8 & 5,4 \\
\hline Ninguno & 7 & 4,7 & 0 & 0 & 0 & 0 & 6 & 4 & 0 & 0 & 2 & 1,4 & 15 & 10,1 \\
\hline TOTAL & 43 & 29,1 & 11 & 7,4 & 13 & 8,8 & 52 & 35,1 & 9 & 6,1 & 20 & 13,5 & 148 & 100 \\
\hline
\end{tabular}

En la tabla 3, se muestra los accidentes laborales que manifiestan haber sufrido los trabajadores de salud durante el desarrollo de sus actividades, existiendo un preocupante $29,1 \%$ de trabajadores que han sufrido pinchazos, por material punzo cortante, así como el 33,1 \% manifiesta haber sufrido la salpicadura de algún tipo de material biológico como son las muestras de sangre, de orina y de heces de los pacientes, siendo el personal técnico en enfermería el más expuesto (23,7 \% del total). Existe una relación significativa entre el tipo de accidentes laborales y el personal y/o trabajador de salud con $\mathrm{p}<0,05$. 
Contrastación del manejo interno de residuos sólidos con la norma técnica del MINSA

Tabla 4. Etapas del manejo interno de residuos sólidos hospitalarios según la calificación de la Norma Técnica N 096-2012-MINSA/DIGESA-V.01, hospital "Víctor Ramos Guardia”, Huaraz, 2013

\begin{tabular}{|c|c|c|c|c|c|c|c|c|c|c|}
\hline \multirow{3}{*}{ ETAPAS } & \multicolumn{8}{|c|}{ CALIFICACIÓN } & \multirow{2}{*}{\multicolumn{2}{|c|}{ TOTAL }} \\
\hline & \multicolumn{2}{|c|}{ Satisfactorio } & \multicolumn{2}{|c|}{ Aceptable } & \multicolumn{2}{|c|}{ Deficiente } & \multicolumn{2}{|c|}{$\begin{array}{l}\text { Muy Defi- } \\
\text { ciente }\end{array}$} & & \\
\hline & $\mathrm{N}^{\circ}$ & $\%$ & $\mathrm{~N}^{\circ}$ & $\%$ & $\mathrm{~N}^{\circ}$ & $\%$ & $\mathrm{~N}^{\circ}$ & $\%$ & $\mathrm{~N}^{\circ}$ & $\%$ \\
\hline Acondicionamiento & 27 & 31,1 & 35 & 40,2 & 24 & 27,6 & 1 & 1,1 & 87 & 100 \\
\hline Almacenamiento primario & 16 & 18,4 & 29 & 33,3 & 42 & 48,3 & 0 & 0 & 87 & 100 \\
\hline Segregación & 5 & 5,7 & 18 & 20,7 & 15 & 17,2 & 49 & 56,4 & 87 & 100 \\
\hline Almacenamiento intermedio & 12 & 13,8 & 21 & 24,1 & 37 & 42,5 & 17 & 19,6 & 87 & 100 \\
\hline $\begin{array}{c}\text { Transporte o recolección } \\
\text { interna }\end{array}$ & 27 & 31,1 & 40 & 46 & 19 & 21,8 & 1 & 1,1 & 87 & 100 \\
\hline Almacenamiento central & 9 & 10,3 & 26 & 29,9 & 50 & 57,5 & 2 & 2,3 & 87 & 100 \\
\hline Tratamiento & 18 & 20,8 & 41 & 47,1 & 23 & 26,4 & 5 & 5,7 & 87 & 100 \\
\hline Disposición final & 21 & 24,1 & 44 & 50,6 & 18 & 20,7 & 4 & 4,6 & 87 & 100 \\
\hline
\end{tabular}

En la presente tabla se observa, que acondicionamiento (40,2 \%), el transporte o recolección interna (46\%), el tratamiento $(47,1 \%)$ y la disposición final (50,6 \%), obtuvieron el calificativo de "satisfactorio"; mientras que el almacenamiento primario, almacenamiento intermedio, y el almacenamiento central fueron calificados como "deficientes" con el 48,3 \%, 42,5 \% y 57,5 \%, respectivamente, desconociendo a donde van a parar los residuos sólidos del hospital. Asimismo, fue considerado como muy "deficiente" la segregación (56,4\%), es decir no se estaría separando y clasificando dichos residuos en el punto de generación, siendo esto último preocupante ya que se estarían combinado los residuos peligrosos con los comunes, siendo esto un potencial riesgo de contaminación para el personal de limpieza del hospital. 
Grado de relación entre el manejo de los residuos sólidos y el riesgo laboral

Tabla 5. Manejo interno de residuos sólidos según el riesgo laboral, hospital Víctor Ramos Guardia, Huaraz, 2013

\begin{tabular}{|c|c|c|c|c|c|c|c|c|}
\hline \multirow{3}{*}{$\begin{array}{l}\text { MANEJO INTERNO DE } \\
\text { RESIDUOS SÓLIDOS }\end{array}$} & \multicolumn{6}{|c|}{ RIESGO LABORAL } & \multirow{2}{*}{\multicolumn{2}{|c|}{ TOTAL }} \\
\hline & \multicolumn{2}{|c|}{ Alto riesgo } & \multicolumn{2}{|c|}{ Mediano riesgo } & \multicolumn{2}{|c|}{ Bajo riesgo } & & \\
\hline & $\mathrm{N}^{\circ}$ & $\%$ & $\mathrm{~N}^{\circ}$ & $\%$ & $\mathrm{~N}^{\circ}$ & $\%$ & $\mathrm{~N}^{\circ}$ & $\%$ \\
\hline Satisfactorio & 7 & 8,1 & 13 & 14,9 & 8 & 9,2 & 28 & 32,2 \\
\hline Aceptable & 14 & 16,1 & 26 & 29,9 & 0 & 0 & 40 & 46 \\
\hline Deficiente & 6 & 6,9 & 5 & 5,7 & 4 & 4,6 & 15 & 17,2 \\
\hline Muy Deficiente & 1 & 1,1 & 1 & 1,2 & 2 & 2,3 & 4 & 4,6 \\
\hline TOTAL & 28 & 32,2 & 45 & 51,7 & 14 & 16,1 & 87 & 100 \\
\hline
\end{tabular}

En la presente tabla, se da a conocer la calificación del manejo interno de residuos sólidos", según el riesgo laboral al que están expuestos los trabajadores asistenciales, evidenciándose que el mayor porcentaje del personal asistencial considera que el manejo de dichos residuos es "aceptable" y que a la vez presentan un "mediano riesgo" laboral en sus actividades diarias con un $29,9 \%$ del total general.

\section{DISCUSIÓN}

Los resultados de la presente investigación nos indican que la calificación del manejo interno de los residuos hospitalarios, por parte de los trabajadores asistenciales, es aceptable con un $46 \%$ del total general, estos no concuerdan con los hallazgos realizados por Curro (2007) quien concluye que, en tres hospitales de Ica, el manejo de los residuos hospitalarios es de baja calidad y deficiente. En otro estudio realizado por Soto y Olano (2004) en un hospital de Chiclayo se concluyó que existe un alto grado de conocimiento de las normas de bioseguridad por el personal profesional y técnico de enfermería; sin embargo, el cumplimiento de las normas de bioseguridad es en promedio de nivel 2 (30 a $60 \%$ ).

En relación al riesgo laboral al que están expuesto los trabajadores de salud del hospital Víctor Ramos Guardia (tabla 2), en los resultados encontrados, un poco más de la mitad de los trabajadores de salud (51,7 \%), identifica un riesgo laboral medio; mientras que $32,2 \%$ de dichos trabajadores está expuesto a un peligroso alto riesgo durante sus actividades diarias, no existiendo las medidas de protección necesarias para su manejo. 
Respecto a los accidentes laborales, un preocupante 29,1\% de trabajadores han sufrido pinchazos, por material punzo cortante, así como el 33,1\% manifiesta haber sufrido la salpicadura de algún tipo de material biológico como son las muestras de sangre, de orina y de heces de los pacientes, seguido por las cortaduras con un 16,9\% de los casos, siendo el personal técnico en enfermería el más expuesto (23,7\% del total), todo esto evidencia la vulnerabilidad y condiciones poco propicias en las cuales se encuentra trabajando el personal del hospital Víctor Ramos Guardia, esto podría conllevar a que existan mayores riesgos como los identificados por la Organización Mundial de la Salud en el 2010, donde se encontró que la administración de inyecciones en condiciones no seguras llegó a causar 33, 800 nuevas infecciones por VIH, 1,7 millones de infecciones por el virus de la hepatitis B y 315. 000 infecciones por el virus de la hepatitis C. (OMS, 2013).

Debemos tener en cuenta que un sujeto que experimenta un pinchazo con una aguja previamente utilizada en un paciente, existe el riesgo de infección por el VHB, el VHC y el VIH en un $30 \%, 1,8 \%$ y 0,3 \%, respectivamente (Bell DM, 1997).

En este sentido, el análisis estadístico de la presente investigación concluyó que existe una relación significativa entre las variables con $\mathrm{p}<0,05$. Resultados similares, pero en porcentajes mayores han obtenido los siguientes investigadores: Vera (2012) concluyó que el área de vigilancia epidemiológica y control de infecciones registraron pinchazos en un $40 \%$ del total.

Con referencia a la contrastación del manejo interno de los residuos sólidos según la Norma Técnica de Gestión y manejo de residuos sólidos en establecimientos del Ministerio de Salud (MINSA), se observa que el acondicionamiento adecuado obtuvo el calificativo de aceptable (40,2\%), el transporte o recolección interna: 46 \%, tratamiento: 47,1 \% y la disposición final: 50,6 \%. En este mismo tema Díaz (2014) en su investigación encontró que el tratamiento de los residuos sólidos hospitalarios en la ciudad de Trujillo es deficiente; mientras que en el almacenamiento primario, almacenamiento intermedio, y el almacenamiento final fueron calificados como deficientes con el 48,3 $\% ; 42,5 \% ; 57,5 \%$ respectivamente, esto se evidencia también en la investigación de Curro (2007), donde indica que el almacenamiento primario y el almacenamiento final es muy deficiente; asimismo, fue considerado como muy "deficiente" la segregación (56,4 \%), es decir no se estaría separando y clasificando dichos residuos en el punto de generación, siendo esto último preocupante ya que se estarían combinado los residuos peligrosos con los comunes, siendo esto un potencial riesgo de contaminación para el personal de limpieza del hospital, esto último coincide con los hallazgos de los siguientes investigadores: Changoluisa y Real (2010), indican que la segregación presenta un nivel deficiente, debido a la mezcla de los desechos comunes e infecciosos sin previo tratamiento. Mahdi y otros (2006) nos indican que la falta de separación entre 
peligrosos y residuos no peligrosos, la ausencia de las reglas y reglamentos necesarios para la aplicación de la recogida de los residuos de las salas del hospital, el transporte, el lugar de almacenamiento temporal, la falta de tratamiento adecuado de los residuos y eliminación de desechos hospitalarios, junto con basura municipal, fueron los principales hallazgos.

Respecto al grado de relación entre el manejo de los residuos sólidos y el riesgo laboral al que están expuestos los trabajadores de salud del hospital Víctor Ramos Guardia el mayor porcentaje de los encuestados indica que el manejo de los residuos sólidos es "aceptable" y que a la vez presentan un "mediano riesgo" en sus actividades diarias $(29,9 \%)$.

En conclusión los análisis estadísticos permitieron encontrar una relación significativa entre el manejo interno de residuos sólidos y el riesgo laboral, con $\mathrm{p}<0,05$, rechazándose la hipótesis nula aceptándose la hipótesis de investigación. Los mismos resultados fueron hallados por Lengua, Junchaya y Quispe (2014), que en su investigación se evidencia una relación directa moderada significativa entre las barreras de protección que aplica el profesional de Enfermería y la Exposición al Riesgo Químico, relación inversa baja pero no significativa entre las Barreras de Protección que aplica el Profesional de Enfermería y la Exposición al Riesgo biológico y relación inversa mínima pero no significativa entre las Barreras de Protección que aplica el Profesional de Enfermería y la Exposición al Riesgo físico.

\section{CONCLUSIONES}

En términos generales queda demostrado que existe una relación significativa entre el manejo inadecuado de residuos sólidos y el riesgo laboral al que están expuestos los trabajadores asistenciales de salud del hospital Víctor Ramos Guardia de Huaraz.

El manejo interno de los residuos sólidos, es aceptable según el $46 \%$ de los trabajadores, resultando preocupante que un significativo 17,2\% presenta deficiencias con respecto a este manejo, evidenciándose que el mayor porcentaje corresponde al personal técnico en enfermería.

Los riesgos laborales son altos, respecto al manejo de residuos sólidos hospitalarios, pues $32,2 \%$ de trabajadores está expuesto a un peligroso alto riesgo durante sus actividades diarias y el $(51,7 \%)$ dice tener un riesgo laboral medio, siendo los riesgos biológicos a los que se encuentran más expuestos, como es el caso de las salpicaduras de sustancias orgánicas debido a la manipulación de las muestras de sangre (28 \%), y las muestras de orina de los pacientes (10\%), existe un preocupante 29,1\% de trabajadores que han sufrido pinchazos, en especial el personal técnico en enfermería con el 10,2\%; seguido por las cortaduras con el 16,9\% de casos, la conducta a seguir es 
lavarse las manos con agua y jabón (68,8 \%), y solo el $31.2 \%$ notifica inmediatamente el accidente laboral.

Referente a la contrastación del manejo interno de los residuos sólidos con la norma técnica de gestión y manejo de residuos sólidos en establecimientos de salud y servicios médicos de apoyo del MINSA, se observa, que solo el transporte o recolección interna en el hospital Víctor Ramos Guardia, obtuvo un calificativo de "satisfactorio" con el 31,1 \%; mientras que el acondicionamiento, tratamiento y disposición final fueron calificados como "aceptables" con el 40,2 \%; 47,1 \% y 50,6 \%, respectivamente, y "deficientes" el almacenamiento primario (48,3\%) y el almacenamiento intermedio (42,5\%), siendo la segregación "muy deficiente" con el 56,4\%. Los resultados del análisis estadístico permitieron encontrar una relación significativa entre el manejo interno de residuos sólidos y el riesgo laboral, con $\mathrm{p}<0,05$.

Al evaluar el grado de relación entre el manejo de los residuos sólidos y el riesgo laboral al que están expuestos los trabajadores de salud, es un manejo "aceptable" de los residuos sólidos y que a la vez presentan un "mediano riesgo" en sus actividades diarias con el $29,9 \%$.

\section{AGRADECIMIENTOS}

A las autoridades de la Universidad Nacional Santiago Antúnez de Mayolo por el apoyo económico para la realización de este trabajo de investigación y a las autoridades del hospital "Víctor Ramos Guardia" y al personal asistencial, por su colaboración desinteresada.

\section{REFERENCIAS BIBLIOGRÁFICAS}

Bell, David. 1997. «Occupational risk of human immunodeficiency virus infection in healthcare workers: an overview». The American Journal of Medicine, Vol. 102, N 5. 9-15. <http:/ /www.sciencedirect.com/science/article/pii/S0002934397894417> [Consulta: 07-05-2016]

Changoluisa, Luis y Real, Luis. 2010. Auditoría de gestión ambiental del manejo de desechos hospitalarios en el hospital Alfredo Noboa Montenegro del cantón Guaranda provincia Bolivar año 2010. Tesis de Licenciatura. Universidad Estatal de Bolívar. Facultad de Ciencias Administrativas, Gestión Empresarial e Informática. Escuela de Gestión Empresarial. Carrera de Contabilidad y Auditoría Cp. <http://dspace.ueb.edu. ec/bitstream/123456789/392/3/tesis\%20auditoria.pdf $>$ [Consulta: 07-06-2013]. 
Curro, Olga. 2007. «Nivel de calidad del manejo de residuos sólidos en hospitales de la provincia de Ica». Académica Perú Salud, Vol. 14, N².94-96 < http:/ /200.62.146.19/ BVRevistas/rev_academia/2007_n2/pdf/a12v14n2.pdf $>$ [Consulta: 08-062013].

Díaz, Delver y Ortiz, Susana. 2014. Mejoramiento del sistema integrado de seguridad, salud en el trabajo y medio ambiente, para reducir los riesgos y el impacto ambiental en la empresa Quiros Rojas Hermanos Contratistas Generales SRL basado en la norma OHSAS 18001: 2007 E ISO 14001:2004. Tesis de grado. Ingeniería Industrial. Universidad privada del Norte <http://repositorio.upn.edu.pe/handle/11537/179> [Consulta: 08-062015].

Lengua Wendy; Junchaya, Silvia y Quispe, Carolina. 2014. «Medidas de bioseguridad que aplica el profesional de enfermería y su relación con la exposición al riesgo laboral en el hospital Santa María del Socorro», Revista enfermería a la vanguardia, Vol. 2, N 1. 10. <http://s3.amazonaws.com/academia.edu.documents/38489301/30-121-1 PB .pdf?AWSAccess KeyId=AKIAIWOWYYGZ2Y53UL3A\&Expires=149544748 6\&Signature $=\mathrm{DaPMknWX} 4 \mathrm{OlhU}$ bz7uxh2AKEQVI\%3D\&response-content disposition=inline $\% 3 \mathrm{~B} \% 20$ filename $\% 3$ DMEDIDAS_DE_BIOSEGURIDAD_ QUE_APLICA_EL_PR.pdf> [Consulta: 09-05-2016].

Mahdi, Farhan y otros. 2006. Estado de la gestión de residuos hospitalarios en irán: un caso estudiar en los hospitales universitarios de la universidad de irán de ciencias médicas. Universidad de Irán de Ciencias Médicas, Teherán, Irán.

Ministerio de Salud. 2004. Procedimientos para el manejo de residuos sólidos hospitalarios. LimaPerú. Chttp://www.disasterinfo.net/PEDSudamerica/leyes/leyes/suramerica/ peru/salud/Resolucion_Ministerial_217.pdf $>$ [Consulta: 09-05-2013].

Organización Mundial de la Salud. 2013. Desechos de las actividades de atención sanitaria. <www.who.int/mediacentre/factsheets/fs253/es> [Consulta: 09-05-2013].

Ortiz Morales, Ignacio. 2010. Diagnóstico situacional sobre el manejo de los residuos peligrosos biológico infecciosos (RPB) en el personal de intendencia de un Centro de Salud TIII de la ciudad de México. Tesis de maestría. Escuela nacional de medicina y homeopatía. México D.F. <http://www.enmh.ipn.mx/PosgradoInvestigacion/Documents/ tesismsosh/ORTIZMORALESIGNACIOCARLOS.pdf $>$ [Consulta: 09-052013].

Soto, Victor y Olano, Enrique. 2004. «Conocimiento y cumplimiento de medidas de bioseguridad en personal de enfermería. Hospital Nacional Almanzor Aguinaga. Chiclayo 2002». Anales de la Facultad de Medicina, Vol. 65, N² 2. 103-110. UNMSM. Facultad de Medicina. <http://www.scielo.org.pe/scielo.php?script=sci_ arttext\&pid=S1025-55832004000200004 > [Consulta: 09-05-2013]. 
Vera, Johannes y Romero, Mercedes. 2012. Caracterización del manejo de desechos Hospitalarios infecciosos a través de una Auditoria Ambiental inicial y propuesta de un modelo de gestión para su segregación, transporte almacenamiento y disposición final en el Hospital Teodoro Maldonado Carbo del IESS. Tesis para el Grado de Magister. Universidad Politécnica Salesiana Guayaquil- Ecuador. <http://dspace.ups.edu.ec/bitstream/123456789/3633/1/ UPS-GT000348.pdf> [Consulta: 09-06-2013].

Recibido: $13 / 07 / 17$

Aceptado: 30/10/17

\section{Correspondencia}

Rafael Marcos Norabuena Penadillo rafaelnorabuenap@hotmail.com 